\title{
Multisensor-Configuration for Improved Identifiability and Observability of Electromechanical Motion Systems
}

\author{
Mathias Tantau, Mark Wielitzka, and Tobias Ortmaier \\ Institute of Mechatronic Systems \\ Leibniz University Hannover \\ An der Universität 1, 30823 Garbsen, Germany \\ \{mathias.tantau, mark.wielitzka, tobias.ortmaier\}@imes.uni-hannover.de
}

\author{
Lars Perner \\ Lenze Automation GmbH \\ Am Alten Bahnhof 11, \\ 38122 Braunschweig, Germany \\ lars.perner@lenze.com
}

\begin{abstract}
For many problems in the field of control design phenomenological models are required so that the need for parameter identification of given model structures arises. These models can be combined with observers to derive the system states in operation in addition to the parameters. However, identification and observation are limited in accuracy due to the restriction to existing series sensors. In the case of electric drives it is possible that due to elasticities in the structure part of the system is vibrating while the position sensor measures a nearly constant position. In this paper, the use of additional acceleration sensors is investigated in terms of identifiability and observability, which are installed at different points of the structure. The analysis is traced back to measures on the sensitivity matrix, where the integrating behaviour of the plant and the combination of different sensor types (position, velocity, acceleration) require special consideration. An industry-like stacker crane is used as a testbed for validation. It is shown that both identifiability and observability can be improved by the additional sensors in many cases. There is a good agreement between the expected and measured frequency response when the acceleration sensor is mounted on the first or second mass. Deviations only occur when mounted on the load suspension device, which is assumed to be the third mass.
\end{abstract}

Index Terms-Multisensor System, Degree of Identifiability, Degree of Observability, Integrating Systems, Servo Systems

\section{INTRODUCTION}

For many problems in the areas of controller design, flatness-based control and condition monitoring, process models with a known internal structure and physically meaningful parameters are required (phenomenological models) [1]. These models can be supplemented by observers which estimate the states of the system at runtime.

In the case of electromechanical motion systems offline parameter identification and online state observation are severely limited in accuracy if only series sensors are used. This is often only one current sensor and one position sensor. This allows an elastically coupled multiple-mass system to carry out vibrations that can hardly be captured by the sensors, especially in systems with high gear ratios. The corresponding states and parameters can only be determined imprecisely.
In this paper, the use of additional acceleration sensors is investigated, which are installed at different points of the structure. They can be mounted without reference frame and the signals can be transferred wireless. The benefit on identifiability and observability is analysed systematically for a range of multiple-mass systems with sensors in different positions.

Although several papers about optimal sensor placement exist [2], [3], [4], [5], multiple-mass models have been considered only in a few references [6], [7] and the benefit of accelerometers for electric drives has not been shown. In the following it is revised if the known criteria can be applied to the problem at hand.

Identifiability can be analysed structurally [8], [9], [10], [11], [12], [6]. It is the question if the model parameters can be determined with a suitable excitation for (almost) all parameterizations without taking into account the actual excitation and the measurement noise. Extensions to multiple sensors are readily possible.

After structural identifiability has been verified a degree of identifiability should be determined to compare or optimize the sensor configurations. The degree of identifiability is often derived from the singular values of the sensitivity matrix [13], [14], [15], [16]. That is the derivative of the measurements with respect to the parameters. Only some of the criteria can be used to compare different numbers of sensors. For example, in [13] the columns of the sensitivity matrix are normalized to unit length so that adding a sensor will not necessarily improve the measure of identifiability. This is also true for the condition number [17].

Observability of the states becomes important when it is considered to use the accelerometers not only for commissioning but also during normal operation. Observability deals with the question if the initial state of a system can be reconstructed from a finite measurement of the system's input and output. Structural observability of linear models can be evaluated by the help of symbolic calculations with the Kalman, Gilbert or Hautus critierion [18], [19], [20]. Graphical approaches are given in [21], [19]. These criteria can be extended to several sensors but in some cases only 
position sensors can be considered [18].

A degree of observability is usually derived from the observability Gramian matrix or the empirical observability Gramian [2], [22], [23], [5]. However, the Gramian can only be determined if all system poles are restricted to the left half plane. In multiple-mass models of electric drives with integrating characteristic poles exist on the imaginary axis. This special case is not even covered by the generalization of the Gramian to unstable systems given in [24], [25]. In [26] it is argued that a degree of observability can be derived from the Kalman observability matrix, which exists also for integrating and unstable systems. But since this matrix contains merely the basis vectors with different physical units and not the matrix that is actually inverted to calculate the initial state, only a coarse assessment of observability is possible.

In the following the different criteria are applied to a class of multiple-mass systems considering the challenges as explained before.

\section{ASSESSMENT OF IDENTIFIABILITY AND OBSERVABILITY}

In this section the methodology for assessing identifiability and observability is explained along with the combinatorial generation of multiple-mass systems for modelling electric drives.

\section{A. Identifiability}

The problem of identifiability is divided into structural identifiability and a degree of identifiability. Before these two are explained, the cost function for identification is defined because it determines largely the identifiability.

1) Cost function for the parameter identification: The identification is performed in frequency-domain because for linear systems this allows an assessment of the identifiability of transfer function models almost independent of the exact excitation. Often, the cost function consists of the distance in the complex plane for each frequency between model and measurement. However, according to [27] for the identification of multiple-mass models better convergence is achieved with the difference in the logarithmic amplitudes in the cost function. The frequency response function (FRF) is written as a vector $\boldsymbol{G}_{\mathrm{v}}$ with one element for each frequency of each scalar transfer function of the MIMO system. For a number of $N_{\mathrm{f}}$ frequencies, $N_{\mathrm{i}}$ inputs and $N_{\mathrm{o}}$ outputs this vector contains $N_{\mathrm{f}} N_{\mathrm{i}} N_{\mathrm{o}}$ elements. The measured FRF is written as $\boldsymbol{G}_{\mathrm{vM}}$. This leads to the following cost function:

$$
J=\left\|\left[\begin{array}{c}
\pi\left(\log _{10}\left\{\left|\boldsymbol{G}_{\mathrm{v}}(\boldsymbol{p})\right|\right\}-\log _{10}\left\{\left|\boldsymbol{G}_{\mathrm{vM}}\right|\right\}\right) \\
\angle\left\{\boldsymbol{G}_{\mathrm{v}}(\boldsymbol{p})\right\}-\angle\left\{\boldsymbol{G}_{\mathrm{vM}}\right\}
\end{array}\right]\right\|_{2}^{2} .
$$

$\boldsymbol{p} \in \mathbb{R}^{N_{\mathrm{p}}}$ is the parameter vector. The weighting factor $\pi$ ensures that $20 \mathrm{~dB}$ difference in the amplitude has an equivalent effect as $180^{\circ}$ phase difference. This cost function is used for the subsequent identifiability analysis.
2) Structural identifiability: Models that can be described by a transfer function matrix $\boldsymbol{G}(s)$ with

$$
G_{i j}(s)=\frac{b_{m, i j} s^{m}+\cdots+b_{1, i j} s^{1}+b_{0, i j}}{s^{n}+a_{n-1, i j} s^{n-1}+\cdots+a_{1, i j} s^{1}+a_{0, i j}}
$$

can be analysed for structural identifiability with the help of the transfer function matrix approach, also called Laplace transform approach [28]. Note that the transfer function is normalized so that the leading coefficient in the denominator is 1 . The other coefficients are functions of the model parameters $\boldsymbol{p}$. Writing the coefficients into a function $\boldsymbol{y}=\boldsymbol{f}(\boldsymbol{p})$ global structural identifiability is equivalent to the fact that a unique solution exists for $\boldsymbol{p}$ with respect to $\boldsymbol{y}$. Local structural identifiability is given if the matrix $\partial \boldsymbol{f} / \partial \boldsymbol{p}$ has full column rank. Here, only the sufficient criterion for local structural identifiability is evaluated.

3) Sensitivity and degree of identifiability: The sensitivity matrix corresponding to the cost function (1) is derived by calculating the derivative of each element of (1) with respect to the parameters:

$$
\begin{gathered}
\boldsymbol{S}=\left[\begin{array}{c}
\boldsymbol{S}_{\mathrm{A}} \\
\boldsymbol{S}_{\mathrm{P}}
\end{array}\right], \\
\boldsymbol{S}_{\mathrm{A}, k j}=\frac{\pi / \ln (10)}{\left|\boldsymbol{G}_{\mathrm{v}, k}\right|^{2}}\left[\operatorname{Im}\left\{\boldsymbol{G}_{\mathrm{v}, k}\right\} \boldsymbol{S}_{\mathrm{i}, k j}+\operatorname{Re}\left\{\boldsymbol{G}_{\mathrm{v}, k}\right\} \boldsymbol{S}_{\mathrm{r}, k j}\right], \\
\boldsymbol{S}_{\mathrm{P}, k j}=\frac{1}{\left|\boldsymbol{G}_{\mathrm{v}, k}\right|^{2}}\left[\operatorname{Re}\left\{\boldsymbol{G}_{\mathrm{v}, k}\right\} \boldsymbol{S}_{\mathrm{i}, k j}-\operatorname{Im}\left\{\boldsymbol{G}_{\mathrm{v}, k}\right\} \boldsymbol{S}_{\mathrm{r}, k j}\right]
\end{gathered}
$$

with the expressions

$$
\begin{gathered}
\boldsymbol{S}_{\mathrm{r}}=\frac{\mathrm{dRe}\left\{\boldsymbol{G}_{\mathrm{v}}(\boldsymbol{p})\right\}}{\mathrm{d} \boldsymbol{p}}=\operatorname{Re}\left\{\frac{\mathrm{d} \boldsymbol{G}_{\mathrm{v}}(\boldsymbol{p})}{\mathrm{d} \boldsymbol{p}}\right\}, \\
\boldsymbol{S}_{\mathrm{i}}=\frac{\mathrm{d} \operatorname{Im}\left\{\boldsymbol{G}_{\mathrm{v}}(\boldsymbol{p})\right\}}{\mathrm{d} \boldsymbol{p}}=\operatorname{Im}\left\{\frac{\mathrm{d} \boldsymbol{G}_{\mathrm{v}}(\boldsymbol{p})}{\mathrm{d} \boldsymbol{p}}\right\} .
\end{gathered}
$$

The right side of (6) and (7) is useful in practice because it allows to calculate the gradient symbolically, while $\operatorname{Re}\{\}$ and $\operatorname{Im}\{\}$ for real and imaginary part are calculated numerically after the frequencies and parameters have been inserted. It can easily be shown that it is allowed to exchange the derivative operator with the $\operatorname{Re}\{\}$ or $\operatorname{Im}\{\}$ operator because the imaginary unit $i$ can be treated as a constant factor in the derivative calculation.

The identifiability of different sensor configurations can be compared by looking at the eigenvalues of $\tilde{\boldsymbol{S}}^{\mathrm{T}} \tilde{\boldsymbol{S}}$. The matrix $\tilde{\boldsymbol{S}}$ is equal to $\boldsymbol{S}$ but with all columns multiplied by the size of the corresponding parameter, as recommended in [13]. If different sensors are used it is usually recommendable to also normalize the measurements to the unit of the respective sensor. However, this is not necessary here, because it can be seen from equation (3) that the sensitivity is independent of a constant scaling of the transfer function, which will affect $\boldsymbol{G}_{\mathrm{v}}$ in the same way as $\boldsymbol{S}_{\mathrm{r}, k j}, \boldsymbol{S}_{\mathrm{i}, k j}$. For the same reason the type of sensor (position, velocity, acceleration) is irrelevant 
for the sensitivity calculation and the results obtained here can equally be applied to e.g. gyroscopes in rotary systems.

In order to measure the identifiability with a single scalar the smallest eigenvalue or the trace of $\tilde{\boldsymbol{S}}^{\mathrm{T}} \tilde{\boldsymbol{S}}$ can be used in the multiple sensor case, because these two measures are axiomatically consistent in the sense that the identifiability of a system with two sensors is at least as good as the identifiability of the two partitioned systems together [26]. This would not be fulfilled for e.g. the largest eigenvalue. This is clear from the fact that $\tilde{\boldsymbol{S}}^{\mathrm{T}} \tilde{\boldsymbol{S}}$ is a symmetric positive (semi-)definite matrix. When additional sensors are used, extra lines are included into $\tilde{\boldsymbol{S}}$, or equivalently a sum of symmetric and positive (semi-)definite matrices of size $N_{\mathrm{p}} \times$ $N_{\text {p }}$ is created:

$$
\boldsymbol{A}+\boldsymbol{B}=\boldsymbol{C} .
$$

Sorting the eigenvalues of matrix $\boldsymbol{A}$

$$
\alpha_{1} \geq \alpha_{2} \geq \cdots \geq \alpha_{N_{\mathrm{p}}}
$$

and similarly for $\beta_{i}$ and $\gamma_{i}$, the eigenvalues of $\boldsymbol{B}$ and $\boldsymbol{C}$, the following relations hold [29]:

$$
\begin{aligned}
\gamma_{\mathrm{N}_{\mathrm{p}}-\mathrm{i}-\mathrm{j}} & \geq \alpha_{N_{\mathrm{p}}-i}+\beta_{N_{\mathrm{p}}-j}, \\
\gamma_{\mathrm{i}+\mathrm{j}-1} & \leq \alpha_{i}+\beta_{j}, \\
\Sigma_{i} \alpha_{i}+\Sigma_{i} \beta_{i} & =\Sigma_{i} \gamma_{i} .
\end{aligned}
$$

From the first, resp. the third relation it is clear that the smallest eigenvalue and the trace are axiomatically consistent measures. From the second relation it follows that the largest eigenvalue is not. It can also be followed from the first relation that adding a sensor will never lower any eigenvalue. However, this property would be lost if a combined measure such as the condition number was used or if the length of each column of the sensitivity matrix was normalized to unit length, as suggested in [13]. Further relations for the sum of eigenvalues can be found in [29].

4) Combinatory generation of multiple mass systems: Modelling electric drives as multiple mass systems a number of different configurations can be distinguished depending on the position of dominant elasticities, sensors and actors. In the following all multiple-mass systems for a given number of elastically coupled masses in a chain are created. The investigation of identifiability will be applied to these systems for different positions of accelerometers. One such system with three masses is shown in Fig. 1. Although translational elements are displayed, rotary elements work equivalently.

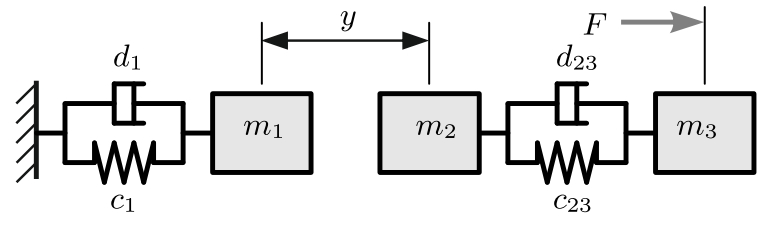

Fig. 1. Example of multiple mass model with $N_{\mathrm{B}}=3, n_{\text {gap }}=1$, $n_{\text {act }+}=3, n_{\text {act }-}=0, n_{\text {meas }+}=2, n_{\text {meas }-}=1$
Apart from actuator and sensor there is always a gap between two masses without spring or damper representing the slideway of the system. Its position is defined by masses $n_{\text {gap }} \in \mathbb{N}$ and $n_{\text {gap }}+1$, in Fig. 1 between masses 1 and 2 .

Actuator and position sensor are located between masses $n_{\text {act }-} \in \mathbb{N}$ and $n_{\text {act }} \in \mathbb{N}$, resp. $n_{\text {meas }-} \in \mathbb{N}$ and $n_{\text {meas }} \in \mathbb{N}$. 0 represents the environment. By varying these indices all possible models can be constructed. In order to ensure that actuators and sensors span around the gap and that the systems are structurally controllable and structurally observable the following rules must be maintained:

$$
\left\{\begin{array}{l}
0 \leq n_{\text {gap }}<N_{\mathrm{B}}, \\
0 \leq n_{\text {act }-} \leq n_{\text {gap }}, \\
n_{\text {gap }}+1 \leq n_{\text {act }+} \leq N_{\mathrm{B}}, \\
0 \leq n_{\text {meas }-} \leq n_{\text {gap }}, \\
n_{\text {gap }}+1 \leq n_{\text {meas }+} \leq N_{\mathrm{B}}, \\
n_{\text {gap }}=0 \vee n_{\text {act }-}>0, \\
n_{\text {gap }}=0 \vee n_{\text {meas }-}>0 .
\end{array}\right.
$$

Additionally, all systems should be excluded that can be created by renaming/renumbering the elements of other systems, see [30], leading to the following additional constraints:

$$
\left\{\begin{array}{l}
n_{\text {act }+} \leq n_{\text {meas }+}, \\
n_{\text {act }+} \leq n_{\text {gap }}+\left\lceil\left(N_{\mathrm{B}}-n_{\text {gap }}\right) / 2\right\rceil .
\end{array}\right.
$$

\lceil\rceil stands for rounding up to integers. In Fig. 1 these two last rules are violated.

Table I holds the numbers of possible multiple-mass systems for a given number of masses $N_{\mathrm{B}}$. Only one position sensor is considered and one actuator. The accelerometers will be added independent of the position sensors later.

TABLE I

NUMBER OF POSSIBLE MODELS FOR DIFFERENT NUMBERS OF MASSES

\begin{tabular}{c|ccccc} 
Masses $N_{\mathrm{B}}$ & 1 & 2 & 3 & 4 & 5 \\
\hline Number of models & 1 & 3 & 11 & 29 & 73
\end{tabular}

In section III the eleven 3-mass systems are analysed for identifiability with and without additional accelerometers. These eleven systems are given in Tab. II. Collocated systems, i.e. systems with identical sensor and actuator position, are marked with an asterisk *.

\section{B. Observability}

Observability is also investigated for all possible 3-mass systems in section III, but the number of systems to consider for a given number of masses is smaller as will be explained in section II-B.2. A degree of observability will be derived in section II-B.1. It is not necessary to check for structural observability because this is ensured by the rules (11), 
TABLE II

ELEVEN 3-MASS MODELS TO CONSIDER FOR IDENTIFIABILITY. THE LETTER OF THE ID REPRESENTS THE POSITION OF THE GAP AND THE ASTERISK * MARKS COLLOCATED SYSTEMS.

\begin{tabular}{l|ccccc} 
System ID & $n_{\text {gap }}$ & $n_{\text {act }-}$ & $n_{\text {act }+}$ & $n_{\text {meas }-}$ & $n_{\text {meas }+}$ \\
\hline A1* & 0 & 0 & 1 & 0 & 1 \\
A2 & 0 & 0 & 1 & 0 & 2 \\
A3 & 0 & 0 & 1 & 0 & 3 \\
A4* & 0 & 0 & 2 & 0 & 2 \\
A5 & 0 & 0 & 2 & 0 & 3 \\
B1* & 1 & 1 & 2 & 1 & 2 \\
B2 & 1 & 1 & 2 & 1 & 3 \\
C1* & 2 & 1 & 3 & 1 & 3 \\
C2 & 2 & 1 & 3 & 2 & 3 \\
C3* & 2 & 2 & 3 & 2 & 3 \\
C4 & 2 & 2 & 3 & 1 & 3
\end{tabular}

meaning that all systems are observable, except possibly for special parameterizations.

1) Degree of observability: The multiple-mass systems can be described by the MIMO state space form:

$$
\dot{\boldsymbol{x}}=\boldsymbol{A}_{\mathrm{S}} \boldsymbol{x}+\boldsymbol{B}_{\mathrm{S}} \boldsymbol{u}, \quad \boldsymbol{y}=\boldsymbol{C}_{\mathrm{S}} \boldsymbol{x}+\boldsymbol{D}_{\mathrm{S}} \boldsymbol{u} .
$$

$\boldsymbol{x} \in \mathbb{R}^{N_{\mathrm{S}}}$ is the states vector, $\boldsymbol{u} \in \mathbb{R}^{N_{\mathrm{i}}}$ is the input vector and $\boldsymbol{y} \in \mathbb{R}^{N_{\text {o }}}$ is the output vector. This also defines the size of the matrices, which can be formed from mass-, stiffness- and damping-matrix, see [31]. The system matrix of all systems considered here contains two eigenvalues at 0 because the force input is integrated twice for the position. In addition it contains eigenvalues related to the flexible vibrations.

In order to obtain the initial states from the measurement of input and output the following relation must be evaluated for a number of time instants [19]:

$$
\boldsymbol{y}(t)=\boldsymbol{C}_{\mathrm{S}} \mathrm{e}^{\boldsymbol{A}_{\mathrm{S}} t} \boldsymbol{x}_{0}+\boldsymbol{C}_{\mathrm{S}} \int_{0}^{t} \mathrm{e}^{\boldsymbol{A}_{\mathrm{S}}(t-\tau)} \boldsymbol{B}_{\mathrm{S}} \boldsymbol{u}(\tau) \mathrm{d} \tau .
$$

The term related to the excitation can be set to zero because it can always be calculated upfront and subtracted. Inserting several time instants for $t$ an overdetermined set of equations for $\boldsymbol{x}_{0}$ is created:

$$
\left(\begin{array}{c}
\boldsymbol{y}\left(t_{1}\right) \\
\vdots \\
\boldsymbol{y}\left(t_{\mathrm{M}}\right)
\end{array}\right)=\underbrace{\left(\begin{array}{c}
\boldsymbol{C}_{\mathrm{S}} \mathrm{e}^{\boldsymbol{A}_{\mathrm{S}} t_{1}} \\
\vdots \\
\boldsymbol{C}_{\mathrm{S}} \mathrm{e}^{\boldsymbol{A}_{\mathrm{S}} t_{\mathrm{M}}}
\end{array}\right)}_{\boldsymbol{S}} \boldsymbol{x}_{0} .
$$

Matrix $\boldsymbol{S}^{\mathrm{T}} \boldsymbol{S}$ can be used for the evaluation of observability from the eigenvalues as above. Again the system outputs are not weighted differently, although this time the measurement unit plays a role. It is believed that with SI units such as $\mathrm{m}$ and $\mathrm{m} / \mathrm{s}^{2}$ a reasonable evaluation is still possible.
Regarding the choice of time instants $t_{1}, \cdots, t_{\mathrm{M}}$ it is not possible to capture the entire settling motion because the system is not stable. Instead a predefined time slot can be sampled with equidistant time samples. It starts at $t=0$ and ends after a time period that is in the order of the longest time constant of the system. The sampling time should be chosen such that the shortest time constant can be captured. Measures derived from this choice of $\boldsymbol{S}$ are highly dependent on the time instants and can therefore only serve for comparison, not as an absolute measure. A similar extension to unstable systems, albeit for the empirical observability Gramian, was proposed in [32].

2) Multiple mass systems for observability: Observability is also investigated for the different multiple-mass systems. They can be generated as in section II-A.4 but because of the linear nature of the systems the input plays no role. Accordingly, the actuator position indices do not need to be varied and the number of systems to consider reduces, see table III. The five 3-mass models are given in Tab. IV.

TABLE III

NUMBER OF POSSIBLE MODELS FOR DIFFERENT NUMBERS OF MASSES

\begin{tabular}{c|ccccc} 
Masses $N_{\mathrm{B}}$ & 1 & 2 & 3 & 4 & 5 \\
\hline Number of models & 1 & 2 & 5 & 9 & 16
\end{tabular}

TABLE IV

ELEVEN 3-MASS MODELS TO CONSIDER FOR IDENTIFIABILITY. THE LETTER OF THE ID REPRESENTS THE POSITION OF THE GAP AND THE ASTERISK * MARKS COLLOCATED SYSTEMS.

\begin{tabular}{c|ccc} 
System ID & $n_{\text {gap }}$ & $n_{\text {meas }-}$ & $n_{\text {meas }+}$ \\
\hline A1* & 0 & 0 & 1 \\
A4* & 0 & 0 & 2 \\
B1* & 1 & 1 & 2 \\
C1* & 2 & 1 & 3 \\
C3* & 2 & 2 & 3
\end{tabular}

\section{RESULTS}

\section{A. Experimental setup}

As the results of the observability and identifiability investigation are partly dependent on the model parameters, an on-site stacker crane for storage and retrieval applications is identified as a 3-mass system and its parameters are used in the theoretical investigations, see Fig. 2. The stacker crane has three orthogonal axes out of which only the horizontal axis along the shelf is used in the experiments. Later, the assumption that the hardware setup exists of three separate masses with elastic coupling is verified by placing accelerometers on the structure and measuring the frequency response function. 


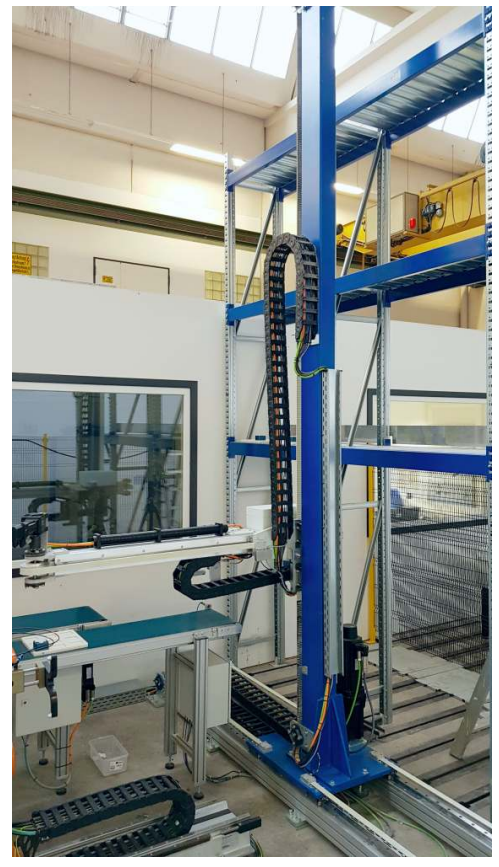

Fig. 2. Experimental testbed stacker crane

Fig. 3 shows the measured FRF and the result of the parameter identification. It is assumed that the 3-mass model A1 is the physically correct model. It can be seen that it is in good agreement with the measurement, except for neglected delay time and a phase difference at low frequencies that might be related to friction and possibly identification in closed loop. The resulting parameters (not shown here) are used as parameterization for the other 3-mass models as well although there is no direct relation to the testbed.

\section{B. Structural identifiability with and without accelerometers}

The result of the analysis of local structural identifiability (see section II-A.2) is given in Tab. V for the eleven 3-mass systems without damping. The calculations are symbolic and parameter values are not required. If the system is identifiable with position sensor only, any combination of additional accelerometers will also be identifiable and no calculation will be required. Otherwise, the right three columns show possible positions for one additional accelerometer. More than one accelerometer has no benefit regarding structural identifiability for systems with three or less masses, but there are a few special cases for four masses where only with two accelerometers identifiability is achieved.

In Tab. VI the same investigation is reported for systems with damping. It can be seen that the damping leads to structural identifiability in most cases.

\section{Degree of identifiability}

The analysis of structural identifiability does not allow a detailed comparison of identifiable sensor configurations.
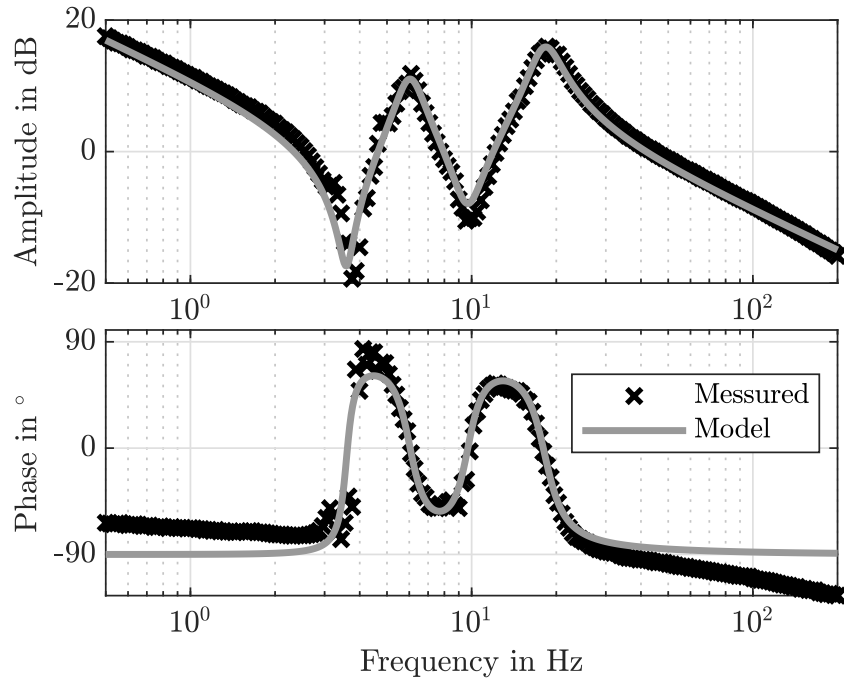

Fig. 3. Measured frequency response function and fitted 3-mass system for motor torque input and velocity output

TABLE V

STRUCTURAL IDENTIFIABILITY OF THE ELEVEN UNDAMPED 3-MASS SYSTEMS WITH POSITION SENSOR ONLY, RESPECTIVELY WITH ADDITIONAL ACCELEROMETER, $\boldsymbol{V}$ : LOCALLY STRUCTURALLY IDENTIFIABLE, $\boldsymbol{X}$ : UNKNOWN, PROBABLY NOT STRUCTURALLY IDENTIFIABLE, -: NO NEED TO CHECK

\begin{tabular}{c|c|ccc} 
System & Only position & \multicolumn{4}{|c}{ Accelerometer at } \\
& sensor & mass 1 & mass 2 & mass 3 \\
\hline A1* & $\checkmark$ & - & - & - \\
\hline A2 & $x$ & $\checkmark$ & $x$ & $x$ \\
\hline A3 & $x$ & $\checkmark$ & $x$ & $x$ \\
\hline A4* & $\checkmark$ & - & - & - \\
\hline A5 & $x$ & $\checkmark$ & $\checkmark$ & $x$ \\
\hline B1* & $\checkmark$ & - & - & - \\
\hline B2 & $x$ & $x$ & $\checkmark$ & $x$ \\
\hline C1* & $\checkmark$ & - & - & - \\
\hline C2 & $x$ & $\checkmark$ & $x$ & $x$ \\
\hline C3* & $\checkmark$ & - & - & - \\
\hline C4 & $x$ & $x$ & $\checkmark$ & $x$ \\
\hline & & & &
\end{tabular}

Especially for the systems with damping further details are required. Therefore, in this section the degree of identifiability is characterized by the smallest eigenvalue, as described in section II-A.3. A frequency range from $1.0 \mathrm{~Hz}$ to $100 \mathrm{~Hz}$ with 200 frequencies, logarithmically spaced is chosen. Again, the results are given with and without damping in Tab. VII and VIII.

As expected, the additional accelerometer can only improve the identifiability but not deteriorate it. In many cases the identifiability is improved considerably, but this is highly dependent on the position of the accelerometer. If it has the 
TABLE VI

STRUCTURAL IDENTIFIABILITY OF THE ELEVEN 3-MASS SYSTEMS, DAMPED, WITH POSITION SENSOR ONLY, RESPECTIVELY WITH ADDITIONAL ACCELEROMETER, $\checkmark$ : LOCALLY STRUCTURALLY IDENTIFIABLE, $\boldsymbol{X}$ : UNKNOWN, PROBABLY NOT STRUCTURALLY IDENTIFIABLE, -: NO NEED TO CHECK

\begin{tabular}{|c|c|c|c|c|}
\hline \multirow[t]{2}{*}{ System } & \multirow{2}{*}{$\begin{array}{c}\text { Only position } \\
\text { sensor }\end{array}$} & \multicolumn{3}{|c|}{ Accelerometer at } \\
\hline & & mass 1 & mass 2 & mass 3 \\
\hline A1* & $\checkmark$ & - & - & - \\
\hline A2 & $\sqrt{ }$ & - & - & - \\
\hline A3 & $x$ & $\sqrt{ }$ & $\sqrt{ }$ & $x$ \\
\hline $\mathrm{A} 4 *$ & $\sqrt{ }$ & - & - & - \\
\hline A5 & $\sqrt{ }$ & - & - & - \\
\hline $\mathrm{B} 1 *$ & $\sqrt{ }$ & - & - & - \\
\hline B2 & $x$ & $x$ & $\sqrt{ }$ & $x$ \\
\hline C1* & $\sqrt{ }$ & - & - & - \\
\hline $\mathrm{C} 2$ & $\sqrt{ }$ & - & - & - \\
\hline $\mathrm{C} 3 *$ & $\sqrt{ }$ & - & - & - \\
\hline $\mathrm{C} 4$ & $\sqrt{ }$ & - & - & - \\
\hline
\end{tabular}

TABLE VII

SMALLEST EIGENVALUE OF THE ELEVEN UNDAMPED 3-MASS SYSTEMS WITH POSITION SENSOR ONLY, RESP. WITH ONE ADDITIONAL ACCELEROMETER

\begin{tabular}{l|c|ccc} 
System & Only position & \multicolumn{3}{|c}{ Accelerometer at } \\
& sensor & mass 1 & mass 2 & mass 3 \\
\hline A1* & 71.9 & 143.7 & 145.8 & 146.7 \\
A2 & 0 & 145.8 & 0 & 0 \\
A3 & 0 & 145.7 & 0 & 0 \\
A4* & 64.4 & 126.2 & 128.7 & 128.3 \\
A5 & 0 & 125.8 & 128.3 & 0 \\
B1* & 73.2 & 142.1 & 146.8 & 146.3 \\
B2 & 0 & 0 & 101.6 & 0 \\
C1* & 51.8 & 143.2 & 60.9 & 75.6 \\
C2 & 0 & 132.2 & 0 & 0 \\
C3* & 0.86 & 94.4 & 4.46 & 0.864 \\
C4 & 0 & 0 & 130.6 & 0
\end{tabular}

same location as the position sensor, the value of the smallest eigenvalue just doubles because as said before all types of sensors have the same effect and accordingly two identical matrices are summed up for $\boldsymbol{A}$ and $\boldsymbol{B}$ in (8). Because of the linearity of eigenvalues, the resulting eigenvalues double. Mostly, the position sensor of the collocated system is located in a comparatively good position and other sensor positions will perform worse. This is, however, different in the case of system $\mathrm{C}^{*}$, where an extra accelerometer on mass 1 is far more effective. It is known from Tab. VI that due to damping systems can become identifiable. But from Tab. VIII it can be seen that the identifiability of these systems is only poor (A2, A5, C2, C4). Here, the improvement achieved with
TABLE VIII

SMALLEST EIGENVALUE OF THE ELEVEN DAMPED 3-MASS SYSTEMS WITH POSITION SENSOR ONLY, RESP. WITH ONE ADDITIONAL ACCELEROMETER

\begin{tabular}{l|c|ccc} 
System & Only position & \multicolumn{3}{|c}{ Accelerometer at } \\
& sensor & mass 1 & mass 2 & mass 3 \\
\hline A1* & 10.9 & 21.7 & 30.6 & 25.8 \\
A2 & 0.006 & 30.6 & 0.013 & 0.010 \\
A3 & 0 & 25.8 & 0.010 & 0 \\
A4* & 3.66 & 12.6 & 7.31 & 6.74 \\
A5 & 0.002 & 11.4 & 6.74 & 0.003 \\
B1* & 11.4 & 17.1 & 12.3 & 12.5 \\
B2 & 0 & 0 & 11.0 & 0 \\
C1* & 0.488 & 2.34 & 5.22 & 0.508 \\
C2 & 0.037 & 2.59 & 0.208 & 0.038 \\
C3* & 0.006 & 3.49 & 0.028 & 0.007 \\
C4 & 0.037 & 0.208 & 1.15 & 0.038
\end{tabular}

accelerometers is particularly obvious.

\section{Degree of observability}

For the analysis of observability a sampling rate of $10 \mathrm{~ms}$ has been chosen and the time duration is set to $0.5 \mathrm{~s}$. The resonance frequencies of the identified model $\mathrm{A} 1 *$ are $6.05 \mathrm{~Hz}$, resp. $18.3 \mathrm{~Hz}$, see Fig. 3. As a result, the degree of observability of the five 3-mass systems is characterized in Fig. 4 by the eigenvalues, as explained in section II-B.1. From left to right the five systems are represented without accelerometer and with accelerometer on masses 1 to 3 . Although only the smallest eigenvalue is an axiomatically consistent measure, the others are also displayed for comparison.

As before, additional sensors cannot lower the eigenvalues and mostly the accelerometers lead to an improvement. An exception is the third mass of the last two systems, where no improvement is visible. These results are a reasonable comparative study, but the exact improvement of the eigenvalues in $\mathrm{dB}$ is not meaningful because it depends on the sensor units. Since these results do not reveal the observability improvement with other types of sensors, this should be investigated further if of interest.

A special case can be found when the system is parameterized symmetric, i.e. the masses are modified so that they are all identical and the same for the stiffness and damping values, while the resonance frequencies are almost unchanged. The result is shown in Fig. 5. Although structurally observable the symmetric parameterization leads to an observability defect of the second system with position sensor only, as also described by [7] for other systems. Consequently, the smallest two eigenvalues are several orders of magnitude smaller. This can be remedied by an accelerometer on mass 1 or 3 . 


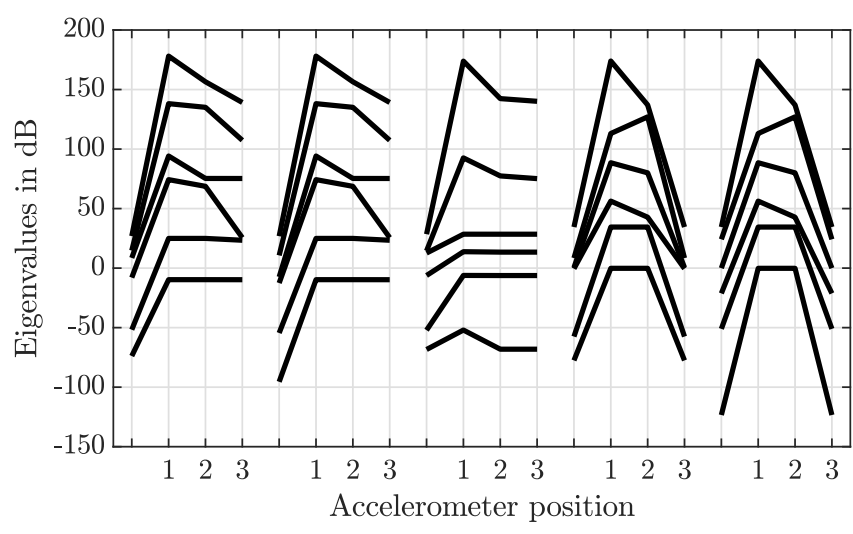

Fig. 4. Eigenvalues of $\boldsymbol{S}^{\mathrm{T}} \boldsymbol{S}$ for the five 3-mass systems representing the observability, RBG parameterization, damped. The abscissa represents also the position of the accelerometer, no number means only the position sensor is used.

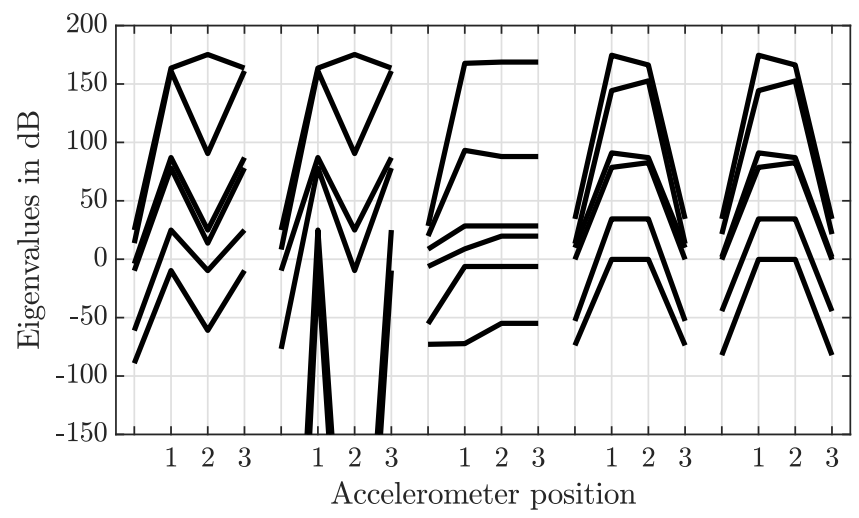

Fig. 5. Eigenvalues of $\boldsymbol{S}^{\mathrm{T}} \boldsymbol{S}$ for the five 3-mass systems representing the observability, symmetric parameterization, damped

\section{E. Accelerometer measurements}

So far, only the parameters have been used from the experimental setup, but no actual experiments have been carried out. In this section, the applicability of the 3-mass model is verified by measuring the FRFs from motor torque to the accelerometers. They are located at what is believed to be the second mass (base of the mast) and third mass (load handling device, close to the mast). The results are shown in Fig. 6 and 7, together with the FRFs expectable from the 3mass model A ${ }^{*}$ with the parameters identified by utilization of the position sensor.

It can be seen that the 3-mass model, which fits accurately for the first mass, see Fig. 3, is still satisfactory for the second mass but cannot predict the measurement at the third mass. A possible explanation is that the mast behaves more like a continuous vibrator rather than a spring between concentrated masses. As a consequence, it is not possible to locate three distinct masses although a clear antiresonance-

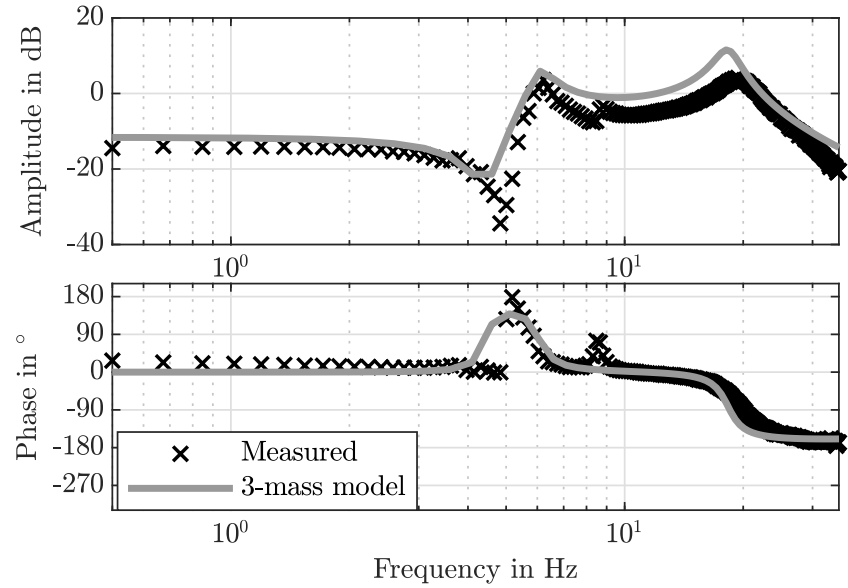

Fig. 6. FRF from torque to acceleration at mass 2, 3-mass model vs. measurement

resonance-antiresonance-resonance behaviour can be seen at the first mass. Attempts to measure at the tip of the mast or at different positions on the load handling device showed slightly different curves but not a better resemblance (not shown here). This limits the utility of accelerometers at the third mass for this testbed despite the theoretical benefit.

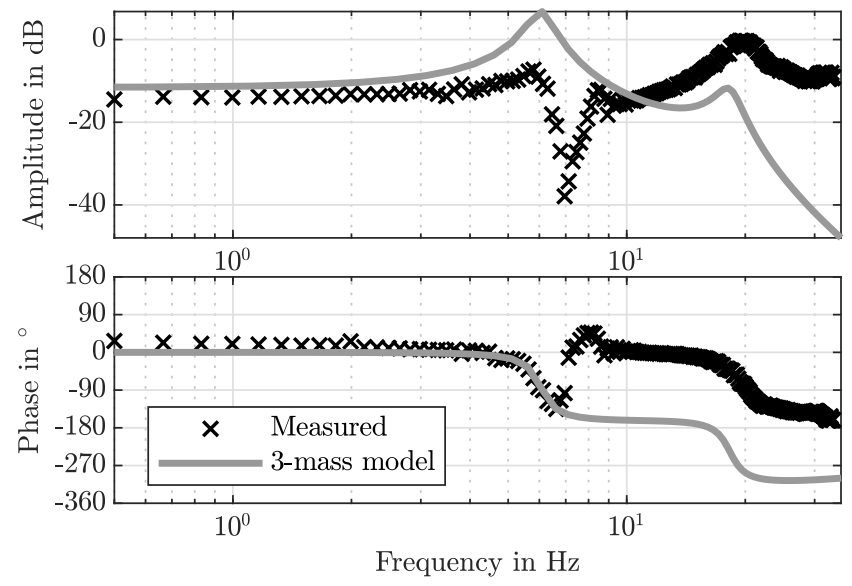

Fig. 7. FRF from torque to acceleration at mass 3, 3-mass model vs. measurement

\section{CONCLUSIONS}

Identifiability and observability of process models are important to many applications of first-principle models, but unfortunately they are often unsatisfactory when only series sensors are used. On the other hand, additional sensors in the form of accelerometers can easily be installed for the time of commissioning at various points of the structure and the question arises to what extend they improve the identifiability and observability. 
In this paper a representative set of multiple-mass models, which characterize a range of electric drives with flexibly coupled mechanics, was investigated. It was shown that structural identifiability can be restored for all models up to three masses by using one additional sensor in a suitable position if it is not given by the position sensor in solitude. Especially, when damping is neglected, the identifiability criterion is often not fulfilled. If damping is considered, structural identifiability is given in a few more cases but it was shown that then the degree of identifiability in terms of the conditioning of the identification problem is poor. In several situations the degree of identifiability can be improved significantly by accelerometers.

Furthermore, it was shown that there is also a benefit for observability, so it could be considered to keep the sensors for normal operation if observability is of interest. In contrast to structural identifiability the type of sensor (position, velocity, acceleration) must be distinguished because the results are different. In experiments with a stacker crane a clear 3-mass resonator behaviour was found but it was not possible to localize three discrete masses, so that a limitation of the identification and observation aided by accelerometers can be seen in deviations of the plaint from the model.

\section{ACKNOWLEDGMENT}

This work was sponsored by the German Forschungsvereinigung Antriebstechnik e.V. (FVA) and the AiF Arbeitsgemeinschaft industrieller Forschungsvereinigungen "Otto von Guericke" e.V.

\section{REFERENCES}

[1] M. Witczak, A. Obuchowicz, and J. Korbicz, "Genetic programming based approaches to identification and fault diagnosis of non-linear dynamic systems," International Journal of Control, vol. 75, no. 13, pp. 1012-1031, 2002.

[2] F. W. J. Van den Berg, H. C. J. Hoefsloot, H. F. M. Boelens, and A. K. Smilde, "Selection of optimal sensor position in a tubular reactor using robust degree of observability criteria," Chemical engineering science, vol. 55, no. 4, pp. 827-837, 2000.

[3] C. Papadimitriou, "Optimal sensor placement methodology for parametric identification of structural systems," Journal of sound and vibration, vol. 278, no. 4-5, pp. 923-947, 2004.

[4] M. Meo and G. Zumpano, "On the optimal sensor placement techniques for a bridge structure," Engineering structures, vol. 27, no. 10, pp. 1488-1497, 2005.

[5] R. Zarringhalam, A. Rezaeian, S. Fallah, A. Khajepour, W. Melek, S.-K. Chen, and B. Litkouhi, "Optimal sensor configuration and faulttolerant estimation of vehicle states," SAE International Journal of Passenger Cars - Electronic and Electrical Systems, 2013.

[6] M. N. Chatzis, E. N. Chatzi, and A. W. Smyth, "On the observability and identifiability of nonlinear structural and mechanical systems," Structural Control and Health Monitoring, vol. 22, no. 3, pp. 574593, 2015.

[7] K. Janschek, Systementwurf mechatronischer Systeme: MethodenModelle-Konzepte. Springer-Verlag, 2010.

[8] R. Bellman and K. J. Åström, "On structural identifiability," Mathematical biosciences, vol. 7, no. 3-4, pp. 329-339, 1970.

[9] H. Pohjanpalo, "System identifiability based on the power series expansion of the solution," Mathematical biosciences, vol. 41, no. 1-2, pp. 21-33, 1978 .
[10] S. Vajda, "Structural equivalence of linear systems and compartmental models," Mathematical Biosciences, vol. 55, no. 1-2, pp. 39-64, 1981.

[11] J. F. M. Van Doren, S. G. Douma, P. M. J. Van den Hof, J. D. Jansen, and O. H. Bosgra, "Identifiability: from qualitative analysis to model structure approximation," International Federation of Automatic Control (IFAC), vol. 42, no. 10, pp. 664-669, 2009.

[12] Q. D. Vu, "Parameter estimation in complex nonlinear dynamical systems," PhDThesis, Technische Universität Ilmenau, 2015.

[13] R. Brun, P. Reichert, and H. R. Künsch, "Practical identifiability analysis of large environmental simulation models," Water Resources Research, vol. 37, no. 4, pp. 1015-1030, 2001.

[14] D. A. Belsley, "A guide to using the collinearity diagnostics," Computer Science in Economics and Management, vol. 4, no. 1, pp. 33-50, 1991.

[15] C. F. Dormann, J. Elith, S. Bacher, C. Buchmann, G. Carl, G. Carré, J. R. G. Marquéz, B. Gruber, B. Lafourcade, P. J. Leitão, et al., "Collinearity: a review of methods to deal with it and a simulation study evaluating their performance," Ecography, vol. 36, no. 1, pp. 27-46, 2013.

[16] A. Gábor, A. F. Villaverde, and J. R. Banga, "Parameter identifiability analysis and visualization in large-scale kinetic models of biosystems," BMC systems biology, vol. 11, no. 1, p. 54, 2017.

[17] G. W. Stewart et al., "Collinearity and least squares regression," Statistical Science, vol. 2, no. 1, pp. 68-84, 1987.

[18] Z.-S. Liu, D.-J. Wang, H.-C. Hu, and M. Yu, "Measures of modal controllability and observability in vibration control of flexible structures," Journal of Guidance, Control, and Dynamics, vol. 17, no. 6, pp. 1377-1380, 1994.

[19] J. Lunze, Regelungstechnik 2, 6th ed. Springer, 1996, vol. 6.

[20] J. Adamy, Nichtlineare Systeme und Regelungen, 2nd ed. Springer, 2014

[21] Y.-Y. Liu, J.-J. Slotine, and A.-L. Barabási, "Observability of complex systems," Proceedings of the National Academy of Sciences, vol. 110, no. 7, pp. 2460-2465, 2013.

[22] A. K. Singh and J. Hahn, "Sensor location for stable nonlinear dynamic systems: Multiple sensor case," Industrial \& engineering chemistry research, vol. 45, no. 10, pp. 3615-3623, 2006.

[23] M. Serpas, G. Hackebeil, C. Laird, and J. Hahn, "Sensor location for nonlinear dynamic systems via observability analysis and maxdet optimization," Computers \& Chemical Engineering, vol. 48, pp. 105-112, 2013.

[24] K. Zhou, G. Salomon, and E. Wu, "Balanced realization and model reduction for unstable systems," International Journal of Robust and Nonlinear Control: IFAC-Affiliated Journal, vol. 9, no. 3, pp. 183-198, 1999.

[25] H. R. Shaker and M. Tahavori, "Optimal sensor and actuator location for unstable systems," Journal of Vibration and Control, vol. 19, no. 12, pp. 1915-1920, 2012.

[26] P. C. Müller and H. I. Weber, "Analysis and optimization of certain qualities of controllability and observability for linear dynamical systems," Automatica, vol. 8, no. 3, pp. 237-246, 1972.

[27] F. Schütte, Automatisierte Reglerinbetriebnahme für elektrische Antriebe mit schwingungsfähiger Mechanik. Shaker, 2003.

[28] C. Cobelli and J. J. Distefano 3rd, "Parameter and structural identifiability concepts and ambiguities: a critical review and analysis," American Journal of Physiology-Regulatory, Integrative and Comparative Physiology, vol. 239, no. 1, pp. R7-R24, 1980.

[29] W. Fulton, "Eigenvalues, invariant factors, highest weights, and schubert calculus," Bulletin of the American Mathematical Society, vol. 37, no. 3, pp. 209-249, 2000.

[30] L.-Q. Zhang, J. C. Collins, and P. H. King, "Indistinguishability and identifiability analysis of linear compartmental models," Mathematical biosciences, vol. 103, no. 1, pp. 77-95, 1991.

[31] M. Tantau, M. Wielitzka, and T. Ortmaier, "Structure and parameter identification of process models with hard non-linearities for industrial drive trains by means of degenerate genetic programming," in ICINCO, vol. 2, Praque, Czech Republic, 2019, pp. 368-376.

[32] M. B. Saltik, L. Özkan, S. Weiland, and P. M. Van den Hof, "Sensor configuration problem: application to a membrane separation unit," International Federation of Automatic Control (IFAC), vol. 49, no. 7, pp. 189-194, 2016. 